\title{
L'infection à chlamydia au Canada de 2010 à 2015
}

\author{
Y Choudhri', J Miller ${ }^{1}$, J Sandhu' ${ }^{1}$ A Leon ${ }^{1}$, J Aho ${ }^{1 \star}$
}

\section{Résumé}

Contexte : La chlamydiose est l'infection transmissible sexuellement à déclaration obligatoire la plus fréquemment rapportée au Canada. Le nombre de cas déclarés annuellement augmente régulièrement depuis 1997.

Objectif : Résumer les tendances observées quant au nombre de cas d'infections à chlamydia au Canada pour la période allant de 2010 à 2015.

Méthodes : Les provinces et territoires canadiens déclarent à l'Agence de la santé publique du Canada tous les cas de chlamydia confirmés en laboratoire. Ainsi, le taux d'infection à chlamydia a pu être calculé à l'échelle nationale, de même que par sexe, par groupe d'âge et par province ou territoire.

Résultats : En 2015, 116499 cas de chlamydia ont été déclarés au Canada, ce qui correspond à un taux de 325 cas par 100000 habitants. Entre 2010 et 2015, les deux tiers des personnes infectées étaient de sexe féminin. Cependant, le taux de personnes infectées a crû davantage chez les hommes au cours de cette période. En 2015, c'est chez les jeunes adultes de 15 à 29 ans que le taux d'infection était le plus élevé. Une augmentation de ce taux a été observée dans tous les groupes d'âges, mais c'est chez les adultes de 40 ans et plus que l'augmentation la plus forte a été constatée (51\%) au cours de la période de 2010 à 2015. Le taux de personnes infectées par chlamydia a augmenté dans la plupart des provinces et territoires au cours de cette période, mais c'est dans les Territoires du Nord-Ouest et au Nunavut, au cours de l'année 2015, que le taux le plus élevé a été signalé.

Conclusion : Entre 2010 et 2015, le nombre de personnes infectées par la chlamydia a connu une augmentation de 16,7 \%. C'est chez les femmes et les jeunes adultes que le taux d'inection rapporté est le plus fort. De nombreux facteurs peuvent expliquer cette tendance à la hausse, mais la possibilité d'une réelle augmentation de l'incidence de l'infection n'est pas exclue. Une surveillance continue de la chlamydia et la recherche des causes des changements observés contribueront à mieux orienter la prévention et le contrôle des infections transmissibles sexuellement.

\begin{abstract}
Affiliation
${ }^{1}$ Centre de la lutte contre les maladies transmissibles et les infections, Agence de la santé publique du Canada, Ottawa (Ontario)
\end{abstract}

${ }^{\star}$ Correspondance : josephine. aho@canada.ca

Citation proposée : Choudhri Y, Miller J, Sandhu J, Leon A, Aho J. L'infection à chlamydia au Canada de 2010 à 2015. Relevé des maladies transmissibles au Canada. 2018;44(2):54-60. https://doi.org/10.14745/ ccdr.v44i02a03f

\section{Introduction}

La chlamydiose, infection causée par la bactérie Chlamydia trachomatis, est l'infection transmissible sexuellement (ITS) la plus fréquemment signalée au Canada. On estime à environ 131 millions le nombre de nouveaux cas d'infections à chlamydia dans le monde en 2012, ce qui en fait l'une des ITS les plus communes (1). Si elle n'est pas traitée, elle peut provoquer une arthrite réactionnelle chez l'homme et la femme, ainsi que I'infertilité, le syndrome inflammatoire pelvien, une douleur pelvienne chronique et des grossesses ectopiques chez la femme, et une orchi-épididymite chez l'homme (2). L'infection est transmissible de la mère à l'enfant à la naissance, ce qui peut causer des cas de pneumonie ou de conjonctivite chez les nouveau-nés (2). En outre, elle amplifie la virulence du VIH et la vulnérabilité à ce virus, car elle augmente le nombre de cellules cibles du VIH présentes dans les voies génitales et stimule l'excrétion du VIH (lorsqu'une cellule infectée libère des particules virales susceptibles d'infecter d'autres cellules) $(3,4)$.
Le nombre de cas de chlamydiose a continuellement augmenté depuis 1997 dans la plupart des provinces et territoires. $(3,4)$. Au-delà de l'utilisation de méthodes de dépistage plus sensibles, il est possible que l'augmentation du nombre de cas soit attribuable à une réelle augmentation de l'incidence de l'infection en raison d'une transmission active (2). En fait, la plupart des personnes infectées n'en sont pas conscientes en raison du caractère asymptomatique de la chlamydiose. C'est pour cette raison qu'elle demeure souvent non traitée, ce qui en favorise la propagation parmi les personnes actives sexuellement. Selon l'Enquête canadienne sur les mesures de la santé 2009-2011, l'incidence de l'infection à chlamydia détectée par analyse d'urine chez les Canadiens de 14 à 59 ans était de $0,7 \%(6)$. Toutefois, aucune des personnes dont l'analyse d'urine a révélé une infection à chlamydia n'a déclaré avoir reçu un diagnostic d'ITS. 
L'incidence de la chlamydia est beaucoup plus élevée chez certaines populations. Dans un village autochtone du Nunavut (7), la proportion de personnes atteintes de la chlamydia s'élevait à $11,6 \%$, tandis qu'elle était de $8,6 \%$ chez les jeunes de la rue des zones urbaines du Canada (8). Les approches traditionnelles, comme celle visant à inciter les patients à aviser leurs partenaires, ont donné des résultats mitigés dans le cas de cette infection largement asymptomatique. Cette approche n'a pas réussi à freiner la transmission de l'infection.

Cet article a pour objectif de résumer les tendances observées quant aux taux de cas déclarés d'infection à chlamydia au Canada au cours de la période allant de 2010 à 2015.

\section{Méthodologie}

\section{Sources des données}

Au Canada, la chlamydiose est une maladie à déclaration obligatoire depuis 1991. Les autorités sanitaires des provinces et des territoires transmettent à l'Agence de la santé publique du Canada (ASPC) des données non nominatives sur les cas confirmés en laboratoire, dans le cadre du Système canadien de surveillance des maladies à déclaration obligatoire (SCSMDO) (9). La définition de cas inclue les sérotypes associés à la lymphogranulomatose vénérienne (LGV). Les définitions de cas sont présentées en annexe (10).

Les données fournies lors de la déclaration comprennent le diagnostic, l'âge au moment du diagnostic, l'année du diagnostic, la province ou le territoire où le diagnostic a été effectué, ainsi que le sexe de la personne atteinte. Les données ont été validées auprès des autorités de la province ou du territoire d'où elles proviennent. Des données étaient disponibles pour toutes les provinces et tous les territoires pour la période du 1 1er janvier 2010 au 31 décembre 2015. Elles ont été extraites du SCSMDO au cours du mois de juillet 2017.

\section{Analyse des données}

L'analyse descriptive des données a été effectuée à l'aide du tableau Microsoft Excel. Les taux annuels nationaux de cas déclarés ont été compilés en utilisant le nombre de cas figurant au SCSMDO comme numérateur et l'estimation annuelle de la population selon les données de Statistique Canada comme dénominateur. Les taux spécifiques au groupe d'âge, au sexe et à la province ou au territoire de résidence ont aussi été calculés. Pour toutes les années visées par l'étude, le nombre de cas est rapporté par tranche de 100000 habitants. L'analyse comparative ne fait appel à aucune méthode statistique. L'effet des fluctuations se fait davantage sentir sur les nombres peu élevés. Les taux correspondants doivent donc être interprétés avec prudence. II se peut que les rapports antérieurs présentent des taux différents pour certaines années en raison des délais de communication des données ou de la mise à jour de ces dernières.

\section{Résultats}

Entre 2010 et 2015, le nombre de cas de chlamydia déclarés s'est accru d'année en année, passant de 94719 à 116 499. Le taux d'infection général en 2015 était de 325 cas par 100000 habitants, en hausse de 16,7 \% par rapport à 2010 .

\section{Sexe et âge}

Bien que le taux d'infections déclarées chez les femmes soit demeuré supérieur à celui retrouvé chez les hommes au cours de la période à l'étude, c'est chez les hommes qu'il a connu la hausse la plus marquée. De 2010 à 2015, le taux d'infection à chlamydia s'est accru de $30,8 \%$ chez les hommes (le nombre de cas passant de 190,4 à 249,1 cas par 100000 habitants) et de $9,3 \%$ chez les femmes (le nombre de cas passant de 364,6 à 398,6 par 100000 habitants) (figure 1).

Figure 1 : Taux globaux ${ }^{a}$ et par sexe des cas déclarés de chlamydiose confirmée en laboratoire de 2010 à 2015 au Canada

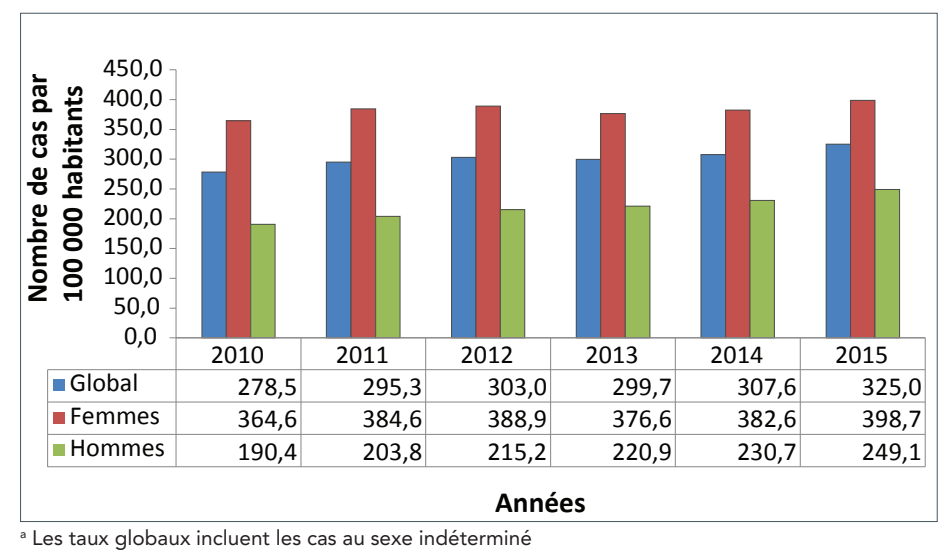

Entre 2010 et 2015, la seule baisse enregistrée du taux de cas déclarés de chlamydiose a été constatée chez les enfants de 10 ans ou moins : il est passé de 1,01 à 0,77 par 100000 habitants (-23,7\%). À l'opposé, la plus forte augmentation du taux de cas déclarés de l'infection, à savoir $51,9 \%$, a été enregistrée chez les adultes de 60 ans et plus (de 4,2 à 6,3 cas par 100000 habitants). Les groupes d'âges de 40 à 59 ans et de 30 à 39 ans suivent avec des augmentations de $51 \%$ et de 40,9\% (de 49 à 74 cas par 100000 habitants et de 263,6 à 371,3 cas par 100000 habitants respectivement). Même si les augmentations les plus fortes ont été enregistrées chez les groupes les plus âgés, les personnes de 60 ans et plus présentaient l'un des taux d'infection absolus les plus bas en 2015 (6,3 par 100000 habitants).

En 2015, le groupe d'âge des 20 à 24 ans présentait le taux d'infection le plus haut avec 1720,4 cas par 100000 habitants. Ce groupe d'âge était le plus affecté à la fois chez les hommes et chez les femmes (figure 2). Les groupes d'âge des 15 à 19 
ans et des 25 à 29 ans suivaient avec des taux d'infection de 1 132,1 par 100000 habitants et de 950,5 par 100000 habitants respectivement. Les adolescents et les jeunes adultes de 15 à 24 ans comptaient pour $56,8 \%$ de tous les cas de chlamydia rapportés en 2015 , alors qu'ils ne représentaient que $12,6 \%$ de la population.

Figure 2 : Taux de chlamydiose confirmée en laboratoire selon le sexe et le groupe d'âge, rapportés en 2015 au Canada

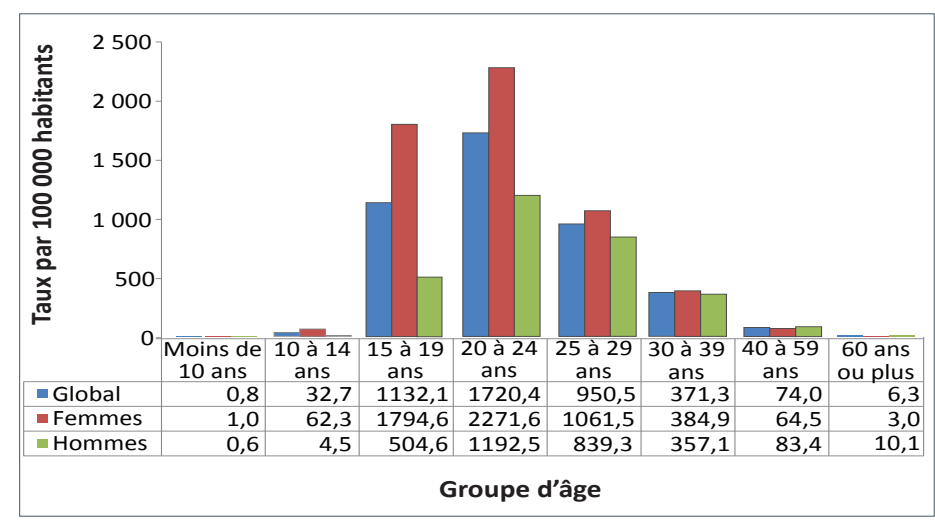

Une augmentation du taux d'infection a été constatée au fil des ans chez les hommes et les femmes de plus de 20 ans. Les augmentations relatives les plus fortes ont été constatées chez les hommes (données non présentées). Au cours de la période de 2010 à 2015, on constate chez les adolescents de 10 à 14 ans une diminution du taux de cas rapportés de 20,7 \% chez les garçons et une augmentation de 9,6\% chez les filles. La tendance est inverse au sein du groupe d'âge des 15 à 19 ans : entre 2010 et 2015 , le taux d'infection a diminué de 0,5\% chez les filles et s'est accru de 19,5\% chez les garçons

\section{Distribution géographique}

Le nombre de cas de chlamydia et le taux de cas déclarés ont augmenté dans la plupart des provinces et territoires au cours de la période de 2010 à 2015 (tableau 1). Toutefois, dans les deux territoires qui présentent les taux d'infection les plus élevés, à savoir les Territoires du Nord-Ouest et le Nunavut, on constate une légère tendance à la baisse au cours de cette période.

Tableau 1 : Nombre de cas et taux de chlamydiose confirmés en laboratoire et signalés, par province et territoire au Canada, de 2010 à 2015

\begin{tabular}{|l|r|r|r|r|r|r|}
\hline \multirow{2}{*}{$\begin{array}{c}\text { Province } \\
\text { ou } \\
\text { territoire }\end{array}$} & \multicolumn{6}{|c|}{$\begin{array}{c}\text { Nombre de cas de chlamydia confirmés } \\
\text { par analyse de laboratoire selon l'année du } \\
\text { diagnostic (taux par 100 000 personnes) }\end{array}$} \\
\cline { 2 - 7 } & $\mathbf{2 0 1 0}$ & $\mathbf{2 0 1 1}$ & $\mathbf{2 0 1 2}$ & $\mathbf{2 0 1 3}$ & $\mathbf{2 0 1 4}$ & $\mathbf{2 0 1 5}$ \\
\hline Alberta & 13112 & 14142 & 15704 & 16081 & 16622 & 17548 \\
& $(351,3)$ & $(373,1)$ & $(404,7)$ & $(402,4)$ & $(404,6)$ & $(419,8)$ \\
\hline Colombie- & 11874 & 11765 & 12416 & 12244 & 13452 & 14379 \\
Britannique & $(265,9)$ & $(261,5)$ & $(273,1)$ & $(266,8)$ & $(289,6)$ & $(306,4)$ \\
\hline Manitoba & 6370 & 6722 & 6589 & 6420 & 6294 & 6539 \\
& $(521,7)$ & $(544,8)$ & $(527,0)$ & $(507,3)$ & $(491,4)$ & $(504,6)$ \\
\hline Nouveau- & 1875 & 1931 & 1932 & 1770 & 1746 & 1891 \\
Brunswick & $(249,0)$ & $(255,6)$ & $(255,3)$ & $(234,2)$ & $(231,3)$ & $(250,7)$ \\
\hline
\end{tabular}

Tableau 1 : Nombre de cas et taux de chlamydiose confirmés en laboratoire et signalés, par province et territoire au Canada, de 2010 à 2015 (suite)

\begin{tabular}{|c|c|c|c|c|c|c|}
\hline \multirow[t]{2}{*}{$\begin{array}{l}\text { Province } \\
\quad \text { ou } \\
\text { territoire }\end{array}$} & \multicolumn{6}{|c|}{$\begin{array}{l}\text { Nombre de cas de chlamydia confirmés } \\
\text { par analyse de laboratoire selon l'année du } \\
\text { diagnostic (taux par } 100000 \text { personnes) }\end{array}$} \\
\hline & 2010 & 2011 & 2012 & 2013 & 2014 & 2015 \\
\hline & $\begin{array}{r}644 \\
(123,4)\end{array}$ & $\begin{array}{r}689 \\
(131,2)\end{array}$ & $\begin{array}{r}864 \\
(164,1)\end{array}$ & $\begin{array}{r}801 \\
(151,9)\end{array}$ & $\begin{array}{r}871 \\
(164,9) \\
\end{array}$ & $\begin{array}{r}963 \\
(182,2) \\
\end{array}$ \\
\hline & $\begin{array}{r}909 \\
(2100,4)\end{array}$ & $\begin{array}{r}825 \\
1896,5) \\
\end{array}$ & $\begin{array}{r}956 \\
2193,0) \\
\end{array}$ & $\begin{array}{r}870 \\
986,9) \\
\end{array}$ & $\begin{array}{r}826 \\
(1882,0) \\
\end{array}$ & $\begin{array}{r}886 \\
(2002,5) \\
\end{array}$ \\
\hline & & & & & & $\begin{array}{r}2835 \\
(300,5)\end{array}$ \\
\hline Nunavut & $\begin{array}{r}1396 \\
(4185,5)\end{array}$ & $\begin{array}{r}1320 \\
(3860,1)\end{array}$ & $\begin{array}{r}1356 \\
(3907,0)\end{array}$ & $\begin{array}{r}1475 \\
(4166,6)\end{array}$ & $\begin{array}{r}1284 \\
(3564,4)\end{array}$ & $\begin{array}{r}1385 \\
(3791,2)\end{array}$ \\
\hline Onta & & & & & & $\begin{array}{l}39024 \\
(282,8)\end{array}$ \\
\hline & $\begin{array}{r}213 \\
(150,3)\end{array}$ & $\begin{array}{r}220 \\
(152,7)\end{array}$ & $\begin{array}{r}257 \\
(177,1)\end{array}$ & $\begin{array}{r}242 \\
(166,7)\end{array}$ & $\begin{array}{r}254 \\
(174,2)\end{array}$ & $\begin{array}{r}227 \\
(154,7)\end{array}$ \\
\hline Québec & & $\begin{array}{l}19147 \\
(239,1) \\
\end{array}$ & $\begin{array}{l}20159 \\
(249,3)\end{array}$ & & & $\begin{array}{l}24448 \\
(296,0)\end{array}$ \\
\hline Sask & $\begin{array}{r}5059 \\
(481,2)\end{array}$ & $\begin{array}{r}5554 \\
(520,8)\end{array}$ & $\begin{array}{r}5721 \\
(526,8)\end{array}$ & $\begin{array}{r}5771 \\
(522,3) \\
\end{array}$ & $\begin{array}{r}5807 \\
(517,9)\end{array}$ & $\begin{array}{r}6091 \\
(538,0)\end{array}$ \\
\hline Yuko & $\begin{array}{r}229 \\
(661,9) \\
\end{array}$ & $\begin{array}{r}209 \\
(590,4) \\
\end{array}$ & $\begin{array}{r}176 \\
(488,1) \\
\end{array}$ & $\begin{array}{r}239 \\
(658,1) \\
\end{array}$ & $\begin{array}{r}210 \\
(569,5) \\
\end{array}$ & $\begin{array}{r}283 \\
(756,8) \\
\end{array}$ \\
\hline Canada & $\begin{array}{l}94719 \\
(278,5)\end{array}$ & $\begin{array}{r}101406 \\
(295,3)\end{array}$ & $\begin{array}{r}105301 \\
(303,0)\end{array}$ & $\begin{array}{r}105347 \\
(299,7)\end{array}$ & $\begin{array}{r}109319 \\
(307,6)\end{array}$ & $\begin{array}{r}116499 \\
(325,0)\end{array}$ \\
\hline
\end{tabular}

\section{Discussion}

De 2010 à 2015 le nombre de cas d'infections à chlamydia déclarés au Canada a augmenté, une tendance similaire à celle observée aux États-Unis (11). Plusieurs facteurs contribuent à expliquer cette augmentation. Outre la possibilité d'une réelle augmentation de l'incidence de l'infection, la popularité d'un nouvel outil diagnostique ultra-sensible, les tests d'amplification des acides nucléiques (TAAN), ainsi que des méthodes de dépistage et de recherche des cas améliorées peuvent avoir entraîné une augmentation du nombre de diagnostics d'infections à chlamydia (12-14). Les autorités sanitaires du Québec, où le taux d'infection a connu l'une des croissances les plus importantes, ont indiqué que ces taux accrus sont en partie attribuables à un dépistage plus systématique et à l'usage croissant des TAAN (15).

Entre 2010 et 2015, selon les cas rapportés, le taux d'infection à chlamydia chez les femmes a été systématiquement plus élevé que chez les hommes, et il continue d'augmenter. Aux États-Unis, en Angleterre et en Australie, on observe également un taux plus élevé chez les femmes $(11,16,17)$. Plusieurs études ont démontré que les femmes, d'un point de vue biologique, pourraient être davantage sujettes à la chlamydiose que les hommes $(18,19)$. De plus, elles sont davantage enclines à solliciter des soins médicaux $(19,20)$. L'infection est souvent asymptomatique chez les femmes. Les infections non traitées peuvent causer une morbidité accrue et entraîner de sérieuses complications comme le syndrome inflammatoire pelvien, des grossesses ectopiques et l'infertilité $(21,22)$. C'est pour cette raison que les programmes de dépistage de la chlamydiose et les médecins ciblent traditionnellement davantage les femmes que 
les hommes (23). Cette tendance de recherche plus fréquente de l'infection chez les femmes peut en partie expliquer les taux d'infection à chlamydia plus élevés observés chez les femmes.

Bien que les taux absolus soient plus bas chez les hommes que chez les femmes, c'est chez les hommes que le taux d'infection a connu la hausse la plus marquée. Cette augmentation peut aussi être en partie attribuable à l'utilisation de méthodes de dépistage plus sensibles, les hommes étant considérés comme une réservoir caché d'infections à chlamydia $(2,24)$. De plus, la plus grande utilisation des TAAN pour détecter les infections autres que génitales (en particulier, les infections rectales chez les hommes ayant des relations sexuelles avec des hommes), ainsi qu'une plus grande disponibilité des tests urinaires d'amplification des acides nucléiques, peuvent expliquer l'augmentation du taux de cas rapportés de chlamydiose chez les hommes (15).

Les adolescents et les jeunes adultes de 15 à 29 ans continuent de présenter un taux élevé de l'infection, une situation observée dans plusieurs pays développés. Par exemple, aux États-Unis, la difficulté d'accès aux soins de santé, des programmes de dépistage déficients, des enjeux de confidentialité lors de la divulgation de pratiques sexuelles risquées ou de partenariats sexuels multiples font partie de l'ensemble de facteurs qui rendent les jeunes plus vulnérables aux ITS (25). Une méconnaissance des risques et l'ignorance des pratiques sécuritaires permettant de les réduire peuvent aussi expliquer cette vulnérabilité aux ITS (26).

Une tendance à la hausse des taux d'infection a aussi été observée chez les cohortes plus âgées. La population canadienne vieillit. Bien que le taux d'infection à chlamydia absolu soit bas chez les personnes de 60 ans et plus, l'incidence de la maladie chez ce groupe d'âge a augmenté de façon notable au cours des dernières années. Il est probable que cette hausse soit attribuable à une combinaison de facteurs, notamment des pratiques sexuelles plus risquées, des changements psychosociaux comme la perte d'un conjoint, l'évolution des normes sociétales, ainsi que des changements physiologiques normaux comme la baisse de la lubrification vaginale qui peut rendre les tissus muqueux plus fragiles et plus sujets aux infections $(27,28)$.

\section{Points forts et limites}

Ce rapport de surveillance présente des données

pancanadiennes sur la chlamydiose, basées sur les cas d'infection signalés par l'ensemble des provinces et territoires du Canada. II décrit le taux de de cas rapportés sur une période de six ans.

Il convient de noter certaines limites inhérentes à ces données. Les données présentées dans ce rapport sous-estiment probablement l'incidence de la chlamydiose au cours de la période de 2010 à 2015 étant donné que de nombreux cas d'infection sont asymptomatiques, ne sont pas dépistés ou ne sont pas déclarées. Le niveau de déclaration peut varier en raison de plusieurs facteurs, notamment une méconnaissance des maladies qu'il est obligatoire de déclarer, le temps nécessaire à leur déclaration et la perception du degré de gravité de la maladie $(29,30)$. Toutefois, dans la mesure où les études $d$ 'incidence et de prévalence entièrement exhaustives sont rares, les données dont nous disposons, issues des cas déclarés, nous fournissent des informations précieuses sur les tendances épidémiologiques de l'infection et sur son taux d'incidence minimal.

Les cas d'infections à chlamydia répétées sont courants. Il est possible qu'une même personne ait été infectée plus d'une fois et qu'à chaque fois, l'infection ait été déclarée. Par conséquent, le nombre réel de personnes infectées pourrait être inférieur au nombre de cas signalés. De plus, le SCSMDO ne comporte pas de données sur les facteurs de risques, ce qui limite notre capacité à cerner les facteurs à l'origine de l'élévation des taux déclarés.

L'absence d'analyse statistique représente aussi une limite de ce rapport. Comme les taux de prévalence n'ont pas été normalisés en fonction de l'âge, il est possible que les changements de taux constatés au cours des trois dernières décennies soient en partie attribuables aux changements de la structure par âge de la population des provinces, des territoires et du Canada en général.

Finalement, les pratiques de dépistage, d'analyse et de déclaration peuvent varier de façon importante selon les provinces et les territoires. Elles peuvent aussi avoir changé avec le temps. Par conséquent, la capacité à dénombrer les cas d'infection n'est pas la même d'un territoire à l'autre ou d'une province à l'autre. Il convient donc de demeurer prudent en effectuant des comparaisons directes entre provinces et territoires.

\section{Conclusion}

Entre 2010 et 2015, le taux de cas de chlamydiose déclarés a connu une hausse de $16,7 \%$. Les taux retrouvés chez les femmes sont systématiquement plus élevés que ceux observés chez les hommes. Même si de nombreux facteurs peuvent expliquer cette tendance à la hausse, la possibilité d'une réelle augmentation de l'incidence de l'infection ne peut être exclue. Une surveillance continue de cette infection, la réévaluation des approches et des méthodes d'intervention traditionnelles et la recherche des causes des changements observés contribueront à mieux orienter la prévention et le contrôle de l'infection à chlamydia.

\section{Déclaration des auteurs}

Y.C. - Conceptualisation, méthodologie, rédaction - première ébauche

J.M. - Logiciel, collecte et conservation des données, validation, analyse formelle, visualisation, rédaction - examen et révision J.S. - Rédaction - première ébauche, visualisation

A.L. - Validation, analyse formelle, visualisation, rédaction examen et révision

J.A. - Conceptualisation, rédaction - première ébauche

\section{Conflit d'intérêt}

Aucun. 


\section{Contributions}

Chris Archibald : supervision, rédaction - examen et révision, ressources, administration du projet

Jennifer Siushansian : rédaction - examen et révision

\section{Remerciements}

Il n'aurait pas été possible de publier le présent rapport sans la contribution continuelle et grandement appréciée de toutes les provinces et tous les territoires à la surveillance nationale des infections transmissibles sexuellement.

\section{Financement}

Le présent travail a été soutenu par l'Agence de la santé publique du Canada.

\section{Références}

1. Newman L, Rowley J, Vander Hoorn S, Wijesooriya NS, Unemo M, Low $\mathrm{N}$ et al. Global estimates of the prevalence and incidence of four curable sexually transmitted infections in 2012 based on systematic review and global reporting. PLoS One 2015 Dec;10(12):e0143304. DOI (http://dx.doi. org/10.1371/journal.pone.0143304). PubMed (https://www. ncbi.nlm.nih.gov/entrez/query.fcgi?cmd=Retrieve \&db=PubMe d\&list_uids=26646541\&dopt=Abstract).

2. Groupe de travail d'experts pour les Lignes directrices canadiennes sur les infections transmissibles sexuellement - Prise en charge et traitement d'infections spécifiques Infections à Chlamydia.In: Wong T, Latham-Carmanico C, rédacteurs/rédactrice. Lignes directrices sur les infections transmissibles sexuellement. Ottawa (ON): Agence de la santé publique du Canada; 2010. https://www.canada.ca/fr/ sante-publique/services/maladies-infectieuses/sante-sexuell e-infections-transmissibles-sexuellement/lignes-directricescanadiennes/infections-transmissibles-sexuellement/ lignes-directrices-canadiennes-infections-transmiss ibles-sexuellement-30.html

3. Galvin SR, Cohen MS. The role of sexually transmitted diseases in HIV transmission. Nat Rev Microbiol 2004 Jan;2(1):33-42. DOI (http://dx.doi.org/10.1038/nrmicro794). PubMed (https:// www.ncbi.nlm.nih.gov/entrez/query.fcgi? cmd=Retrieve\&db=Pu bMed\&list_uids=15035007\&dopt=Abstract).

4. Rebbapragada A, Kaul R. More than their sum in your parts: the mechanisms that underpin the mutually advantageous relationship between HIV and sexually transmitted infections. Drug Discov Today Dis Mech 2007;4(4):237-46. DOI (http:// dx.doi.org/10.1016/j.ddmec.2007.12.003).

5. Totten S, MacLean R, Payne E, Severini A. L'infection à Chlamydia trachomatis et le lyphogranulome vénérien au Canada : 2003-2012. Relevé des maladies transmissibles au Canada. 2015;41(2):22-9. http://www. phac-aspc. gc.ca/publicat/ccdr-rmtc/15vol41/dr-rm41-02/assets/ pdf/15vol41_02-fra.pdf

6. Rotermann M, Langlois KA, Severini A, Totten S. Prevalence of Chlamydia trachomatis and herpes simplex virus type 2: Results from the 2009 to 2011 Canadian Health Measures Survey. Health Rep 2013 Apr;24(4):10-5. https://www.statcan. gc.ca/pub/82-003-x/2013004/article/11777-eng.pdf
7. Steenbeek A, Tyndall M, Sheps S, Rothenberg R. An epidemiological survey of chlamydial and gonococcal infections in a Canadian arctic community. Sex Transm Dis 2009 Feb;36(2):79-83. DOI (http://dx.doi.org/10.1097/ OLQ.0b013e3181898e4d). PubMed (https://www.ncbi.nlm. nih.gov/entrez/query.fcgi?cmd=Retrieve\&db=PubMed\&lis t_uids=19125145\&dopt=Abstract).

8. Shields SA, Wong T, Mann J, Jolly AM, Haase D, Mahaffey $\mathrm{S}$ et al. Prevalence and correlates of Chlamydia infection in Canadian street youth. J Adolesc Health 2004 May;34(5):38490. DOI (http://dx.doi.org/10.1016/S1054-139X(03)00332-X). PubMed (https://www.ncbi.nlm.nih.gov/entrez/query.fcgi?cmd $=$ Retrieve\&db=PubMed\&list_uids=15093792\&dopt=Abstract).

9. Agence de la santé publique du Canada. Système de surveillance des maladies à déclaration obligatoire rapport canadien : 2005-2008. Ottawa (ON): ASPC; 2012. http:// publications.gc.ca/collections/collection_2012/aspc-phac/ HP40-75-2012-fra.pdf

10. Agence de la santé publique du Canada. Définitions nosologiques des maladies transmissibles faisant l'objet d'une surveillance nationale. Relevé des maladies transmissibles au Canada. 2009;35(S2):S1-134. https://www.canada.ca/ content/dam/phac-aspc/migration/phac-aspc/publicat/ ccdr-rmtc/09pdf/35s2-fra.pdf

11. Centers for Disease Control and Prevention. Sexually transmitted disease surveillance 2015. Atlanta (GA): US Department of Health and Human Services; 2016. https:// www.cdc.gov/std/stats15/STD-Surveillance-2015-print.pdf

12. McKay A, Barrett M. Rising reported rates of chlamydia among young women in Canada: what do they tell us about trends in the actual prevalence of the infection? Can J Hum Sex 2008;17(1-2):61-9.

13. Rekart $M L$, Brunham RC. Epidemiology of chlamydial infection: are we losing ground? Sex Transm Infect 2008 Apr;84(2):87-91. DOI (http://dx.doi.org/10.1136/sti.2007.027938). PubMed (https://www.ncbi.nlm.nih.gov/entrez/query.fcgi?cmd=Retrieve $\& d b=$ PubMed\&list_uids=18216155\&dopt=Abstract).

14. Tuite AR, Jayaraman GC, Allen VG, Fisman DN. Estimation of the burden of disease and costs of genital Chlamydia trachomatis infection in Canada. Sex Transm Dis 2012 Apr;39(4):260-7. DOI (http://dx.doi.org/10.1097/ OLQ.0b013e31824717ae). PubMed (https://www.ncbi.nlm. nih.gov/entrez/query.fcgi?cmd=Retrieve $\& d b=P u b M e d \& l i s$ t_uids=22421691\&dopt=Abstract).

15. Blouin K, Venne S, Lambert G. Portrait des infections transmissibles sexuellement et par le sang (ITSS) au Québec: année 2015 (et projections 2016). Québec (QC): INSPQ; 2016. https://www.inspq.qc.ca/en/node/6914

16. The Kirby Institute. HIV, viral hepatitis and sexually transmissible infections in Australia: annual surveillance report 2016. Sydney (AU): University of New South Wales; 2016. https://kirby.unsw.edu.au/sites/default/files/kirby/report/SERP_ Annual-Surveillance-Report-2016_UPD170627.pdf

17. Public Health England. Table 2: New diagnoses \& rates by gender, sexual risk \& age group, 2012-2016. London (UK): Public Health England. https://www.gov.uk/government/ uploads/system/uploads/attachment_data/file/626361/2016_ Table_2_Selected_STI_diagnoses__rates_by_gender_sexual_ risk__age_group.pdf

18. Lee $\mathrm{V}$, Tobin JM, Foley E. Relationship of cervical ectopy to chlamydia infection in young women. J Fam Plann Reprod Health Care 2006 Apr;32(2):104-6. DOI (http://dx.doi.org/1 0.1783/147118906776276440). PubMed (https://www.ncbi. 


\section{SURVEILLANCE}

nlm.nih.gov/entrez/query.fcgi?cmd=Retrieve\&db=PubMed\&li st_uids=16824301\&dopt=Abstract).

19. Wong T, Singh A, Mann J, Hansen L, McMahon S. Gender differences in bacterial STIs in Canada. BMC Womens Health 2004 Aug;4 Suppl 1:S26. DOI (http://dx.doi.org/10.1186/14726874-4-S1-S26). PubMed (https://www.ncbi.nlm.nih. gov/entrez/query.fcgi? $\mathrm{cmd}=$ Retrieve $\& \mathrm{db}=$ PubMed\&lis t_uids=15345089\&dopt=Abstract).

20. Thompson AE, Anisimowicz Y, Miedema B, Hogg W, Wodchis WP, Aubrey-Bassler K. The influence of gender and other patient characteristics on health care-seeking behaviour: a QUALICOPC study. BMC Fam Pract 2016 Mar;17:38. DOI (http://dx.doi.org/10.1186/s12875-016-0440). PubMed (https://www.ncbi.nlm.nih.gov/entrez/query.fcgi?cmd $=$ Retrieve\&db=PubMed\&list_uids=27036116\&dopt=Abstract)

21. Moreno MA, Furtner F, Rivara FP. Advice for patients. Chlamydia screening: a routine test. Arch Pediatr Adolesc Med 2009 Jun;163(6):592 DOI (http://doi.org/10.1001/ archpediatrics.2009.99). PubMed (https://www.ncbi.nlm.nih. gov/pubmed/19487620).

22. Wiesenfeld HC, Solomon CG. Screening for Chlamydia trachomatis infections in women. N Engl J Med 2017 Mar. DOI (http://www.nejm.org/doi/citedby/10.1056/ NEJMcp1412935\#t=article). PubMed (https://www.ncbi.nlm. nih.gov/pubmed/28225683).

23. Machalek K, Hanley BE, Kajiwara JN, Pasquali PE, Stannard CJ. Chlamydia screening practices among physicians and community nurses in Yukon, Canada. Int J Circumpolar Health 2013 Aug;72(1):22447. DOl (http://dx.doi.org/10.3402/ ijch.v72i0.21607). PubMed (https://www.ncbi.nlm.nih. gov/entrez/query.fcgi?cmd=Retrieve\&db=PubMed\&list_ uids $=23984299 \& \mathrm{dopt}=$ Abstract).

24. Barbee LA, Dombrowski JC, Kerani R, Golden MR. Effect of nucleic acid amplification testing on detection of extragenital gonorrhea and chlamydial infections in men who have sex with men sexually transmitted disease clinic patients. Sex Transm Dis 2014 Mar;41(3):168-72. DOI (http://dx.doi.org/10.1097/ OLQ.0000000000000093). PubMed (https://www.ncbi.nlm. nih.gov/entrez/query.fcgi? $\mathrm{cmd}=$ Retrieve\& $\mathrm{db}=$ PubMed\&lis t_uids=24521722\&dopt=Abstract).
25. Centers for Disease Prevention and Control. Sexually transmitted infections among young Americans. Atlanta (GA): US Department of Health and Human Services; 2013. https:// www.cdc.gov/std/products/youth-sti-infographic.pdf

26. Council of Ministers of Education. Canada. Canadian Youth, Sexual Health and HIV/AIDS Study: factors influencing knowledge, attitudes and behaviours. Toronto (ON): Council of Ministers of Education Canada; 2003. http://educ.queensu. $\mathrm{ca} / \mathrm{sites} /$ webpublish.queensu.ca.educwww/files/files/Research/ SPEG/SPEG\%20Canadian\%20Youth\%2C\%20Sexual\%20 Health\%20and\%20HIV.pdf

27. Johnson BK. Sexually transmitted infections and older adults. J Gerontol Nurs 2013 Nov;39(11):53-60. DOl (http://dx.doi. org/10.3928/00989134-20130918-01). PubMed (https://www. ncbi.nlm.nih.gov/entrez/query.fcgi?cmd=Retrieve\&db=PubMe d\&list_uids=24066789\&dopt=Abstract).

28. Poynten IM, Grulich AE, Templeton DJ. Sexually transmitted infections in older populations. Curr Opin Infect Dis 2013 Feb;26(1):80-5. DOI (http://dx.doi.org/10.1097/ QCO.0b013e32835c2173). PubMed (https://www.ncbi.nlm. nih.gov/entrez/query.fcgi?cmd=Retrieve\&db=PubMed\&lis t_uids=23221769\&dopt=Abstract).

29. Friedman SM, Sommersall LA, Gardam M, Arenovich T. Déclaration sous-optimale des maladies à déclaration obligatoire dans les services des urgences au Canada: Enquête sur les connaissances, les pratiques et les obstacles perçus chez les médecins d'urgence. Relevé des maladies transmissibles au Canada. 2006 Sep;32(17):187-98. https:// www.canada.ca/content/dam/phac-aspc/migration/phac-aspc/ publicat/ccdr-rmtc/06pdf/cdr3217.pdf

30. Doyle TJ, Glynn MK, Groseclose SL. Completeness of notifiable infectious disease reporting in the United States: an analytical literature review. Am J Epidemiol 2002 May;155(9):866-74. DOI (http://dx.doi.org/10.1093/ aje/155.9.866). PubMed (https://www.ncbi.nlm.nih. gov/entrez/query.fcgi?cmd=Retrieve $\& \mathrm{db}=$ PubMed\&lis t_uids=11978592\&dopt=Abstract). report 2016. Sydney (AU): University of New South Wales; 2016. https://kirby.unsw.edu. au/sites/default/files/kirby/report/SERP_Annual-SurveillanceReport-2016_UPD170627.pdf 


\section{Annexe : définition des cas confirmés de chlamydia}

Confirmation par analyse de laboratoire d'infections génito-urinaires ou d'infections extragénitales par l'une des trois méthodes suivantes :

- Détection de Chlamydia trachomatis par culture

$\mathrm{OU}$

- Détection de l'acide nucléique de $C$. trachomatis

$\mathrm{OU}$

- Détection d'un antigène de $C$. trachomatis

Confirmation par analyse de laboratoire d'infections périnatales

Détection et confirmation, par l'une des trois méthodes suivantes, de la présence de $C$. trachomatis dans les voies rhino-pharyngiennes ou dans tout échantillon provenant des voies respiratoires d'un nourrisson ayant contracté une pneumonie au cours des six mois suivant la naissance ou dans tout échantillon conjonctival provenant d'un nourrisson ayant contracté une conjonctivite au cours du premier mois suivant la naissance :

- Détection de C. trachomatis par culture

$\mathrm{OU}$

- Détection de l'acide nucléique de $C$. trachomatis

OU

- Détection d'un antigène de $C$. trachomatis

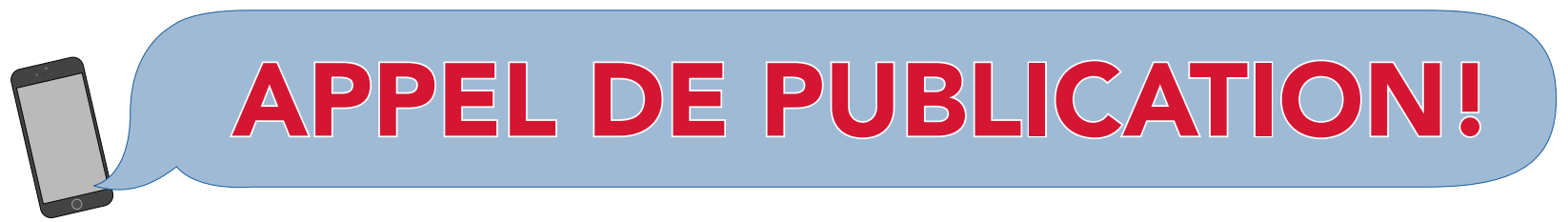

Le RMTC prévoit publier des numéros thématiques dans les domaines suivants et vous invite à soumettre vos textes, en français ou en anglais, au plus tard à la date indiquée ci-dessous :

Thème traité

Hépatite C

Santé rurale

Infections nosocomiales résistantes aux agents antimicrobiens

Sensibilisation à la vaccination
Date limite

12 mars 2018

10 avril 2018

10 juillet 2018

13 août 2018

Rapports d'éclosions - Examens systématiques • Rapports de témoins oculaires Science de l'application des connaissances - Commentaires • Et plus encore

Consultez la page Renseignements à l'intention des auteurs sur le site Web du Relevé des maladies transmissibles au Canada afin d'obtenir de plus amples détails.

Canada.ca/RMTC 